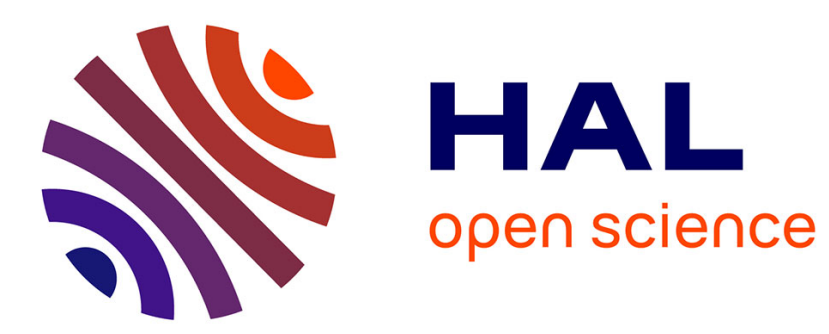

\title{
Hierarchical Modeling of the Mechanical Behavior of High Speed Steels as Layer-Structured Particulate MMCs
}

\author{
A. Plankensteiner, H. Böhm, F. Rammerstorfer, V. Buryachenko
}

\section{- To cite this version:}

A. Plankensteiner, H. Böhm, F. Rammerstorfer, V. Buryachenko. Hierarchical Modeling of the Mechanical Behavior of High Speed Steels as Layer-Structured Particulate MMCs. Journal de Physique IV Proceedings, 1996, 06 (C6), pp.C6-395-C6-402. 10.1051/jp4:1996639 • jpa-00254472

HAL Id: jpa-00254472

https://hal.science/jpa-00254472

Submitted on 1 Jan 1996

HAL is a multi-disciplinary open access archive for the deposit and dissemination of scientific research documents, whether they are published or not. The documents may come from teaching and research institutions in France or abroad, or from public or private research centers.
L'archive ouverte pluridisciplinaire HAL, est destinée au dépôt et à la diffusion de documents scientifiques de niveau recherche, publiés ou non, émanant des établissements d'enseignement et de recherche français ou étrangers, des laboratoires publics ou privés. 


\title{
Hierarchical Modeling of the Mechanical Behavior of High Speed Steels as Layer-Structured Particulate MMCs
}

\author{
A.F. Plankensteiner, H.J. Böhm, F.G. Rammerstorfer and V.A. Buryachenko \\ Institute for Lightweight Structures and Aerospace Engineering, Vienna Technical University, and Christian \\ Doppler Laboratory for Micromechanics of Materials, Vienna Technical University, Gusshausstrasse \\ 27-29, 1040 Vienna, Austria
}

\begin{abstract}
High Speed Steels (HSS) produced by electro-slag remelting can be viewed as particle reinforced Metal Matrix Composites (MMCs) consisting of alternating layers of high and low inclusion volume fraction. These phase arrangements require specific models to be used in analytical and numerical studies of the elastoplastic response of HSS.

In the present study, a hierarchical micro-meso-macro approach is discussed, which combines the Multi-Particle Effective Field Method (MEFM) for describing the matrix-inclusion topology at the microscale with an extended lamination theory for handling the layered geometry at the mesoscale. In addition, a Finite Element based two-dimensional method is presented, in which HSS is modeled as a material with a graded microstructure. The obtained results are discussed in terms of overall elastoplastic behavior and of damage relevant microscale fields.
\end{abstract}

\section{INTRODUCTION}

High Speed Steels (HSS) are materials of considerable practical importance in metals forming, where they are used especially for producing dies as well as cutting tools of complex shape. Typical routes for the production of HSS are powder metallurgical processing and electro slag remelting. After hot forming and heat treatment, HSS produced by the latter approach are characterized by a layered meso structure, in which so-called carbide stringers showing an elevated volume fraction of carbidic inclusions alternate with layers of low inclusion content (see Figure 1a). These particulates, the primary carbides, have characteristic sizes of a few $\mu \mathrm{m}$ and consist of composite metallic carbides. The matrix material is a martensitic steel containing homogeneously distributed nanometer-sized secondary carbides.

In modeling the mechanical behavior of high speed steels, the effects of the secondary carbides can be handled within the constitutive description of the matrix material. The arrangement of the primary carbides, however, must be explicitly accounted for, standard micromechanical methods for particle reinforced metal matrix composites (MMCs) being incapable of describing effects due to the layered structure in a satisfactory way. In the present contribution, two complementary approaches are used to deal with the specific phase arrangement of HSS, one being a semi-analytical micro-meso-macro method using statistical micromechanics and the other a numerical description of MMCs with a graded matrix-inclusion microstructure. 


\section{MICRO-MESO-MACRO MODEL}

The guiding idea behind the micro-meso-macro model is the description of the HSS at two different length scales. At the microlevel, each layer is treated as a particle reinforced MMC of appropriate inclusion volume fraction, the matrix-inclusion microtopology of which is explicitly accounted for. At the meso level the HSS is viewed as consisting of alternating homogenized elastoplastic composite layers which take the roles of the carbidic stringers and the inclusion-poor regions between them.

\subsection{Mesomechanical Modeling}

An elastoplastic extended lamination theory is used to obtain relations between the overall load and the homogenized layer fields, i.e. to link the macro and meso quantities (denoted by two and one overbars, respectively). The thicknesses of the individual layers are taken to be small in comparison

a)

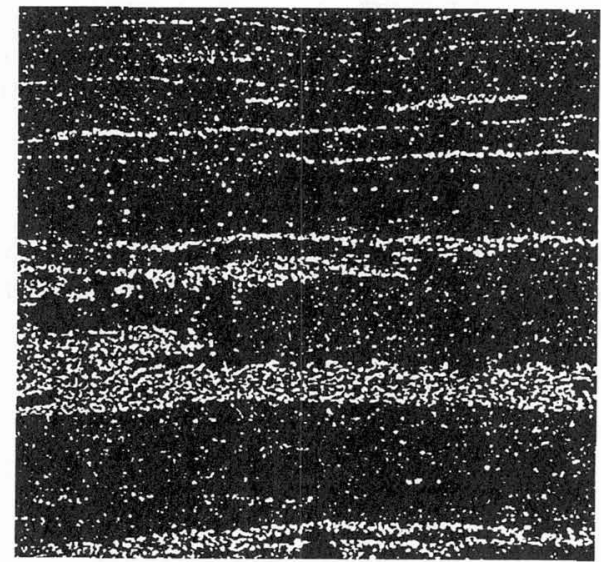

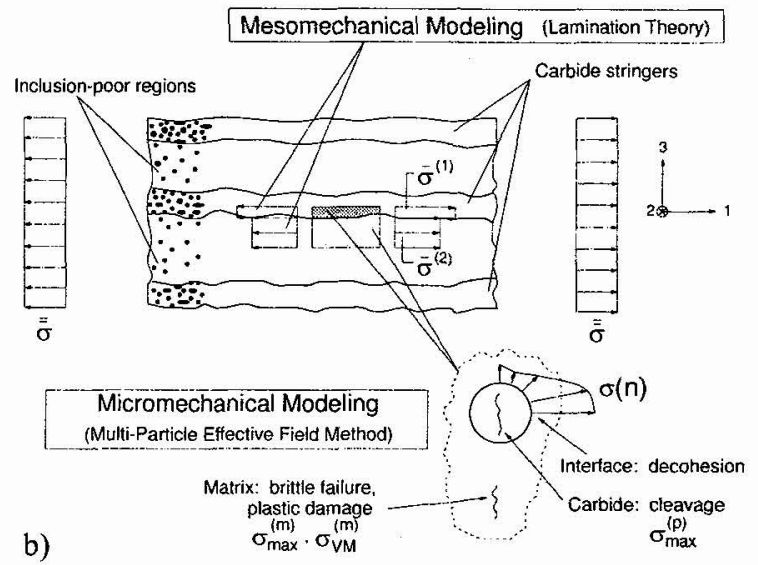

b)

Figure 1: a) Metallograph of an HSS (HS-6-5-2-5) and b) micro-meso-macro mechanical modeling concept for layer structured particulate composites.

to the thickness of the sample, and for the sake of simplicity we assume that the carbide stringers (1) and the regions between them ${ }^{(2)}$ are stacked periodically, see Figure $1 \mathrm{~b}$. The mechanical loading of an individual layer is taken to be due to uniform global stresses acting in the 1-2-plane (in-plane stresses) $\overline{\bar{\sigma}}=\left(\overline{\bar{\sigma}}_{11}, \overline{\bar{\sigma}}_{22}, \overline{\bar{\sigma}}_{12}\right)^{T}$, which give rise to global in-plane strains $\overline{\bar{\varepsilon}}=\left(\overline{\bar{\varepsilon}}_{11}, \overline{\bar{\varepsilon}}_{22}, \overline{\bar{\gamma}}_{12}\right)^{T}$ as well as nonzero global strains in the thickness direction, $\overline{\bar{\varepsilon}}_{33}$.

The meso model is formulated in terms of homogenized layer stresses $\langle\bar{\sigma}\rangle^{(j)}=\left\langle\bar{\sigma}_{11}^{(j)}, \bar{\sigma}_{22}^{(j)}, \bar{\sigma}_{12}^{(j)}\right\rangle^{T}$ and strains $\langle\bar{\varepsilon}\rangle^{(j)}=\left\langle\bar{\varepsilon}_{11}^{(j)}, \bar{\varepsilon}_{22}^{(j)}, \bar{\gamma}_{12}^{(j)}\right\rangle^{T},(j=1,2)$ which are constant over each layer's volume. A plane stress state is assumed at the layer level, i.e. $\left\langle\bar{\sigma}_{i 3}\right\rangle^{(j)}=0,(i=1,2,3 ; j=1,2)$. Each layer is understood to be perfectly bonded to its neighbours and to be characterized by a homogeneous isotropic effective material behavior, which is given in terms of the deformation theory of plasticity. Accordingly, the meso scale material law for the $j$-th layer can be expressed as $\langle\overline{\boldsymbol{\varepsilon}}\rangle^{(j)}=\overline{\mathbf{C}}_{s}^{(j)}\langle\overline{\boldsymbol{\sigma}}\rangle^{(j)},(j=1,2)$ with $\overline{\mathbf{C}}_{s}^{(j)}$ representing the secant compliance matrix. Because the homogenized behavior of elastoplastic MMCs cannot be described satisfactorily by standard metal plasticity theories (e.g. the yield functions of overall MMCs depend also on the first invariant of the stress deviator), the $\overline{\mathbf{C}}_{s}^{(j)}$ must be obtained from a suitable micromechanical theory. 


\subsection{Micromechanical Modeling}

Each layer is treated as a particle reinforced matrix-inclusion composite. The elastoplastic martensitic matrix is assumed to be homogeneous and isotropic. Its isotropic hardening behavior is described by the Ludwik equation $\sigma_{y}=\sigma_{y}^{0}+h\left(\varepsilon^{* p l}\right)^{n}$, where $\sigma_{y}^{0}$ and $\sigma_{y}$ represent the initial yield stress and the actual yield stress, respectively, and $\varepsilon^{* p l}$ stands for the accumulated plastic strain of the matrix. $h$ is the hardening coefficient and $n$ is the hardening exponent of the matrix material. The primary carbides are modeled as spherical inclusions with isotropic purely elastic material behavior. They are assumed to be distributed randomly in the matrix material corresponding to a nearly homogeneous probability density function.

For describing the elastoplastic behavior of the composite material making up each layer, the MultiParticle Effective Field Method (MEFM, [1], [2], [3]) is employed. Applying this statistical averaging technique over a given layer, the phase volume averages of the stress states in the carbides $\langle\sigma\rangle^{(p)}$ and the matrix $\langle\sigma\rangle^{(m)}$ are calculated. In the MEFM, the v. Mises equivalent stresses are evaluated using the second moments of the matrix stress deviator fields, $\sigma_{y}=\sqrt{\frac{3}{2}\left\langle s_{k l} s_{k l}\right\rangle^{(m)}} \geq \sqrt{\frac{3}{2}\left\langle s_{k l}\right\rangle^{(m)}\left\langle s_{k l}\right\rangle^{(m)}}$, so that the inhomogeneous character of the microstresses is taken into account. In addition, the ensemble averages of the stress distributions around the initially (i.e. prior to failure initiation) perfect matrix-inclusion interfaces $\langle\boldsymbol{\sigma}(\mathbf{n})\rangle$ are determined for each layer.

\subsection{Results obtained with the Micro-Meso-Macro Model}

The formalism described above is applied to a layer structured HSS and an HSS with a homogeneous distribution of carbides (i.e. a material having the same total carbide volume fraction, but lacking a layered meso structure). A layer thickness of $20 \mu \mathrm{m}$ and an inclusion volume fraction of $\left.\xi^{(p)}\right)^{(1)}=32 \%$ are chosen for the carbidic stringers, the corresponding data for the inclusion-poor layers being $80 \mu \mathrm{m}$ and $\xi^{(p)^{(2)}}=2 \%$, respectively. This corresponds to an overall carbide volume fraction of $\xi^{(p)}=8 \%$. The material parameters are selected to correspond to an HS-6-5-2-5 at room temperature, the Young's modulus of the matrix being set to $E^{(m)}=210 \mathrm{GPa}$, its Poisson's number to $\nu^{(m)}=0.3$, and its initial yield stress to $\sigma_{y}^{0}=2750 \mathrm{MPa}$. For the hardening coefficient and the hardening exponent of the martensitic matrix, values of $h=1500 \mathrm{MPa}$ and $n=0.5$, respectively, are used. The Young's modulus for the composite metallic carbides is chosen as $E^{(p)}=450 \mathrm{GPa}$ and their Poisson's number as $\nu^{(p)}=0.25$.

The micromechanical quantities calculated by taking into account the layered meso structure can
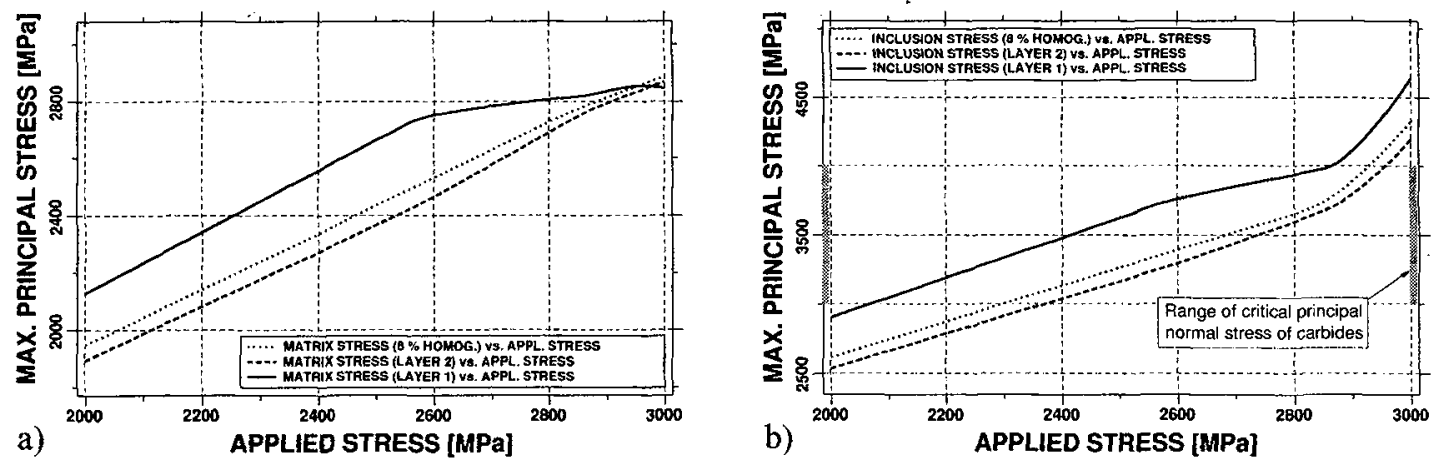

Figure 2: Maximum principal stresses within a) the matrix and b) the carbides, respectively, vs. the applied overall uniaxial tensile stress predicted by the micro-meso-macro approach. 
be compared with appropriate critical values in order to obtain first estimates of the relevant initial failure mechanism at the micro level. For this purpose the predicted maximum principal stresses in matrix and inclusion, which are relevant to brittle failure of the matrix and cleavage of the carbides, respectively, are shown in Figures $2 a$ and $2 b$ in dependence on the applied overall uniaxial tensile stress. Ranges for typical critical values of these principal microstresses are also indicated.

In a similar vein, Figures $3 \mathrm{a}$ and $3 \mathrm{~b}$ display the predicted layer ensemble averages of the maximum
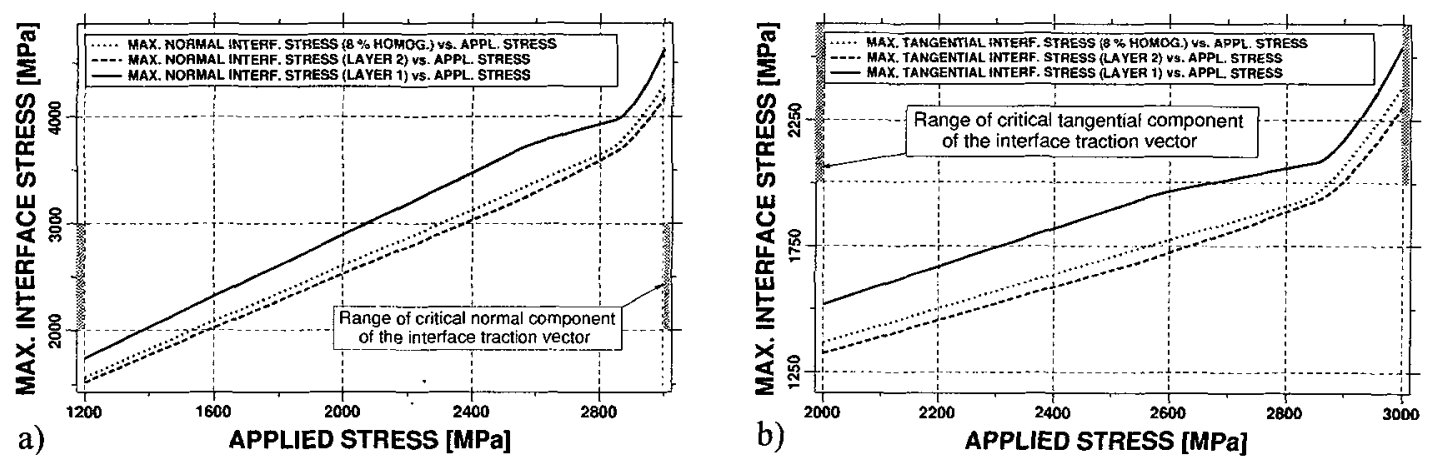

Figure 3: Maximum a) normal and b) tangential components of the interface traction vectors, respectively, vs. the applied overall uniaxial tensile stress predicted by the micro-meso-macro approach.

normal and tangential components of the interfacial tractions, which can be used to evaluate the behavior of the HSS with respect to interfacial decohesion, together with ranges of critical values taken from [7]. A comparison of the different microscale failure modes shows a strong tendency towards normal interfacial decohesion in the carbide stringers, whereas cleavage of carbides plays a noticeable role only if lower cleavage stresses are adopted. Critical parameters for brittle failure of the matrix are not available at this time, which excludes this failure mode from the present considerations. For the chosen range of critical values, tangential decohesion at the interface can generally be excluded as an important initial failure mechanism.

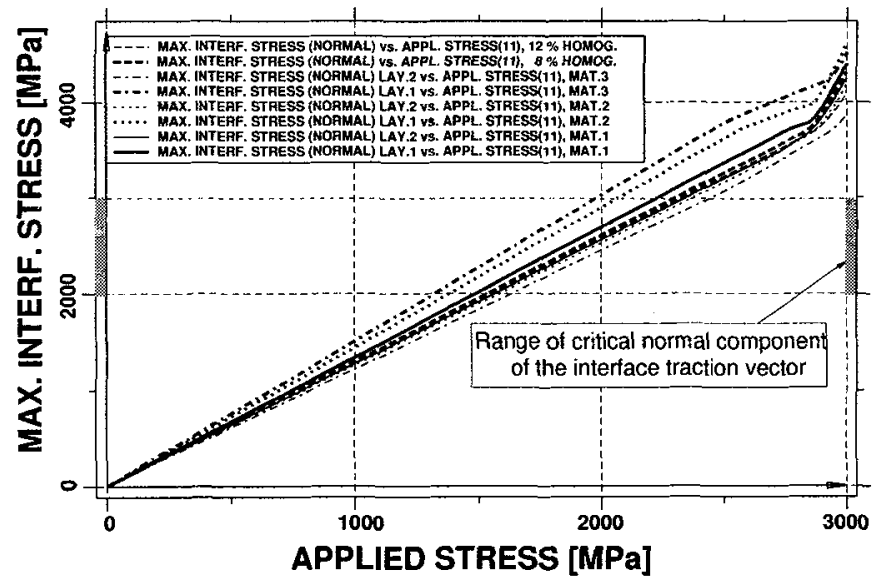

Figure 4: Maximum normal components of the interface traction vectors vs. the applied overall uniaxial tensile stress predicted by the micro-meso-macro approach.
The influence of different carbide stringer topologies on the initiation of normal interfacial decohesion is shown in Figure 4 , where - in addition to the geometrical configuration mentioned above (Material 2) - two further configurations are considered: $\xi^{(p)}=8 \%, \xi^{(p)^{(1)}}=$ $\left.15.31 \%, \xi^{(p)}\right)^{(2)}=3.83 \%$ (Material 1) and $\xi^{(p)}=12 \%, \xi^{(p)}{ }^{(1)}=$ $48 \%, \xi^{(p)^{(2)}}=3 \%$ (Material 3). Furthermore, a homogeneous counterpart to Material 3 $\left(\xi^{(p)}=12 \%\right.$, non-layered homogeneous distribution of carbides) is studied. For all configurations the same constituent properties as for Material 2 are adopted. In the elastic regime the type of the 
layered meso structure shows a significant influence on the interfacial traction. However, during the plastic yielding, which occurs first in the carbide stringers and then in the regions between them, a load transfer between these regions diminishes the influence of the layerwise carbide distribution. It should be noted that the computations started from eigenstress-free (virgin) states. Residual stresses generated by hardening and tempering cycles after hot reduction processes are not yet implemented within the micro-meso-macro model.

\section{HCT MODEL}

\subsection{Concepts of the HCT Model}

An alternative modeling strategy to the micro-meso-macro concept discussed above views the HSS as an MMC with a compositional gradient and uses a Finite Element based periodic microfield approach for describing it. Clearly, unit cells containing a considerable number of inclusions providing for at least some randomness in the positions of the particulates are required for dealing with the complex microgeometries encountered in HSS. At present, computational requirements limit three-dimensional unit cell models of particle reinforced MMCs to cubic arrays (see e.g. [10]) or arrangements of a few particles [9]. With two-dimensional models, in contrast, it is possible to handle arrangements of some.

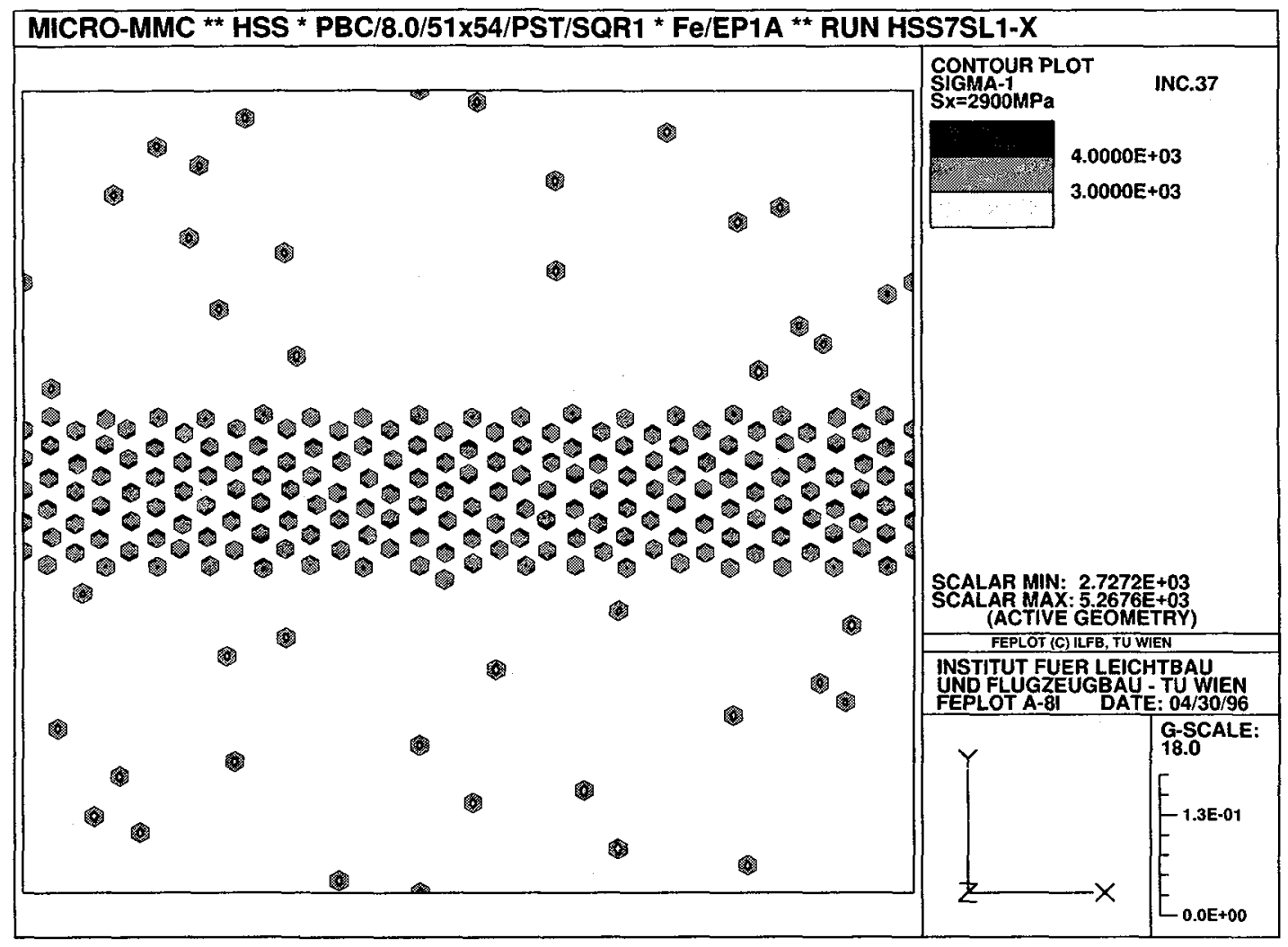

Figure 5: Distribution of maximum principal stresses in the carbidic inclusions predicted by the HCT model for an applied overall uniaxial tensile stress of $2900 \mathrm{MPa}$. 
hundred inclusions, albeit at the cost of some inaccuracies in predicting the mechanical responses of particle reinforced composites (see e.g. [5]), most important of them being a tendency to overpredict the localization of plastic strains.

A relatively simple method for generating two-dimensional phase arrangements consists of tesselating a rectangular computational domain into regular hexagonal cells, each of which is assigned to a phase using deterministic or pseudo-random algorithms. The discretization of the cells and consequently of the phase domains by triangular or quadrilateral elements is rather straightforward. A number of applications of HCT-based studies of inhomogeneous materials are reported in the literature, among them works on particle reinforced MMCs [6] and on duplex steels [8].

The HCT model used in the present work describes a generic microstructure consisting of a band of "single cell" inclusions arranged in a perturbed periodic hexagonal array for describing the carbidic stringers, and some randomly assigned inclusion cells in the other regions. By minor adjustments of the diameters of the "particles" the overall and layer volume fractions selected for Material 2 could be obtained. The material models and parameters for the phases correspond to those employed for the micro-meso-macro model. Periodic boundary conditions were prescribed and plane stress elements were used.

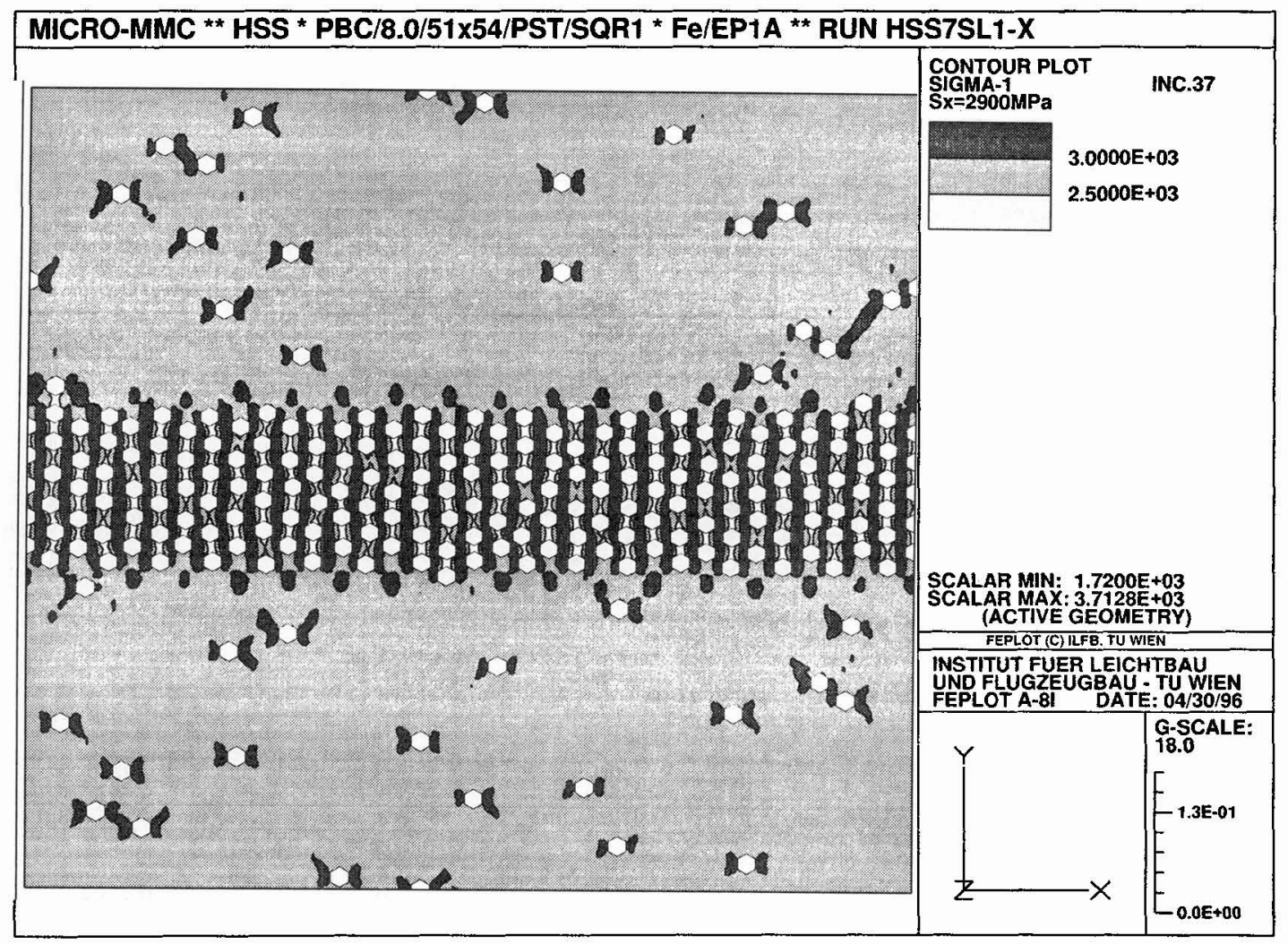

Figure 6: Distribution of maximum principal stresses in the matrix predicted by the HCT model for an applied overall uniaxial tensile stress of $2900 \mathrm{MPa}$. 


\subsection{Results obtained with the HCT Model}

The overall stress-strain curve obtained by the HCT model shows a somewhat more gradual yielding response and a less pronounced hardening behavior compared to the results of the micro-meso-macro approach, the agreement being generally good. All microscale stress results shown in the following correspond to an applied load of $2900 \mathrm{MPa}$, which is unrealistically high for the material in question, but, giving rise to yielding throughout the matrix, can be expected to highlight differences between the micro-meso-macro and HCT models.

The HCT model predicts noticeable fluctuations of the microfields in both phases of the HSS, es-

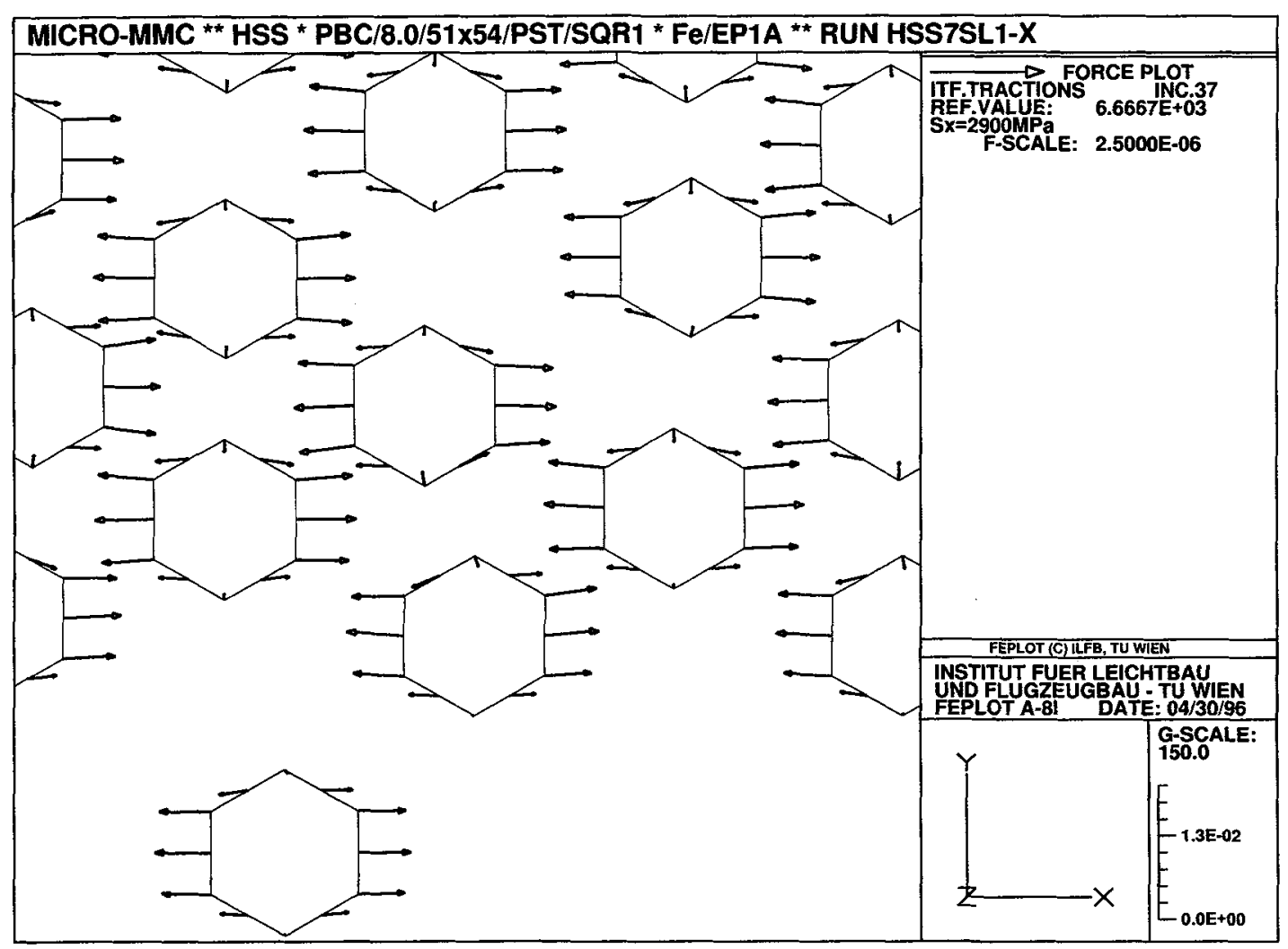

Figure 7: Interfacial traction vectors predicted for an applied overall uniaxial tensile stress of $2900 \mathrm{MPa}$ shown for a detail of the HCT model.

pecially in the inclusion-rich region. Figure 5 shows the predictions for the microscale maximum principal stresses. The corresponding phase averages were evaluated as approximately $3710 \mathrm{MPa}$ and $3240 \mathrm{MPa}$ in the inclusion-rich and inclusion-poor regions, respectively. Maximum values exceeding $5200 \mathrm{MPa}$ are reached locally in inclusions situated in the interior of the carbidic stringers. Analogous results for the maximum principal stresses in the matrix are depicted in Fig. 6, values of up to 3700 $\mathrm{MPa}$ being predicted in the carbide-rich band. The rather regular sequence of highly loaded zones in the stringer region is probably due to an excessively regular microgeometry. Finally, Fig. 7 shows predicted interfacial traction vectors for a detail of the HCT model. Even though at first sight the position dependence of the tractions appears to be rather small, a closer inspection shows systematic trends in the direction of the arrows, which also tend to get longer towards the interior of the stringer, 
where normal tractions of up to $3650 \mathrm{MPa}$ and shear tractions of nearly $2000 \mathrm{MPa}$ are reached. Clearly, the damage relevant parameters considered here show significantly higher levels in the carbidic stringers than in the inclusion-poor regions, which is in good agreement with the view that damage and failure in HSS is initiated predominantly in inclusion rich regions [4]. Also, the values of the microscale maximum principal stresses and interfacial tractions predicted for the layered microgeometry tend to be noticeably higher than those obtained in a companion study using a statistically homogeneous distribution of particles at the same overall inclusion volume fraction of $\xi^{(p)}=8 \%$. These trends are thought to be significant despite the generic character of the HCT analyses.

\section{CONCLUSIONS}

Due to their complementary modeling philosophies and tradeoffs, the two approaches presented in this work allow considerable insight into different aspects of the inelastic behavior of high speed steels. The semi-analytical/statistical micro-meso-macro model provides a computationally efficient method for evaluating the overall behavior as well as the averaged micro quantities in dependence on the layered meso structure. In addition to the geometrical parameters, the influence of material parameters and strength parameters on the mechanical behavior can be studied. By carrying out parameter variations, "critical" configurations with respect to initiation of microscale damage can be detected. The numerical/deterministic model highlights the pronounced inhomogeneity of the microfields in the considered HSS at high loading levels.

Good agreement in the overall results obtained with the two approaches is found. A comparison of the phase averages of the maximum principal stresses, however, shows differences in the predictions for the partitioning of the mechanical loads between the two phases in the plastic range. This is due to the assumption of constant plastic matrix strains within the MEFM, which accordingly predicts a fully plastified matrix immediately after initiation of plastic yielding.

\section{References}

[1] V.A. Buryachenko, Strength Mater. 22 (1990) 1645-1650.

[2] V.A. Buryachenko, W.S. Kreher, J. Mech. Phys. Sol. 43 (1995) 1105-1125.

[3] V.A. Buryachenko, Acta Mech. 119 (1996) 93-117.

[4] H. Fischmeister, J. Paul, S. Karagöz, Pract. Met. 25 (1988) 28-40.

[5] T. Iung, M. Grange, Mater. Sci. Engng. A201 (1995) L1-L8.

[6] P. McHugh, R.J. Asaro, C.F. Shih, Acta metall. mater. 41 (1993) 1461-1510.

[7] M.H. Poech, H.F. Fischmeister, K. Hummert, "Fracture toughness of PM-HSS: The influence of primary carbide distribution", First International High Speed Steel Conference, Leoben, Austria, 26. -28.03.1990, G. Hackl and B. Hribernik Eds. (1990) pp. 73-80.

[8] T. Siegmund, E. Werner, F.D. Fischer, J. Mech. Phys. Sol. 43 (1995) 495-552.

[9] D.F. Watt, X.Q. Xu, D.L. Lloyd, Acta Mater. 44 (1996) 789-799.

[10] E. Weissenbek, H.J. Böhm, F.G. Rammerstorfer, Comput. Mater. Sci. 3 (1994) 263-278. 